\title{
Transverse mode dynamics in vertical-cavity surface-emitting lasers: Spatiotemporal versus modal expansion descriptions
}

\author{
Josep Mulet and Salvador Balle \\ Instituto Mediterráneo de Estudios Avanzados (IMEDEA), Consejo Superior de Investigaciones Científicas-Universitat de les Illes \\ Balears, Campus UIB, E-07071 Palma de Mallorca, Spain
}

(Received 7 May 2002; published 5 November 2002)

\begin{abstract}
We discuss the range of validity of a modal description for the spatiotemporal dynamics of the optical field in vertical-cavity surface-emitting lasers. We focus on the secondary pulsations that appear during the turn-off transients when the injection current is modulated by a square-wave signal. We compare the results obtained with both a full spatiotemporal model [J. Mulet and S. Balle, IEEE J. Quantum. Electron. 38, 291 (2002)] and a modal expansion derived from this model. We find that the results obtained from the two descriptions agree for strong lateral guiding. However, for weak lateral guiding we find differences because the optical-field profile changes significantly due to spatial changes in the refractive index induced by the carrier density. The reason is that in the full spatiotemporal model a shrinkage of the mode profile occurs, which leads to an enhancement of the secondary pulsations. This effect is not included in the modal expansion, and it determines the limits of validity of such an approach for gain-guided devices.
\end{abstract}

DOI: $10.1103 /$ PhysRevA.66.053802

PACS number(s): 42.55.Px, 42.55.Sa, 42.60.Jf

\section{INTRODUCTION}

Vertical-cavity surface-emitting lasers (VCSELs) have received a great deal of attention during recent decades, especially for their potential implementation in optical communication systems. VCSELs present clear advantages over the conventional edge-emitting lasers, such as singlelongitudinal mode emission, circular output beam, low threshold, etc. However, as the injection current is increased, they exhibit instabilities associated with the polarization state of the light and/or the excitation of transverse modes of higher order [2]. These evidences motivate the understanding, characterization, and control of both the polarization and transverse modes in VCSELs. The high complexity of the problem arises from the fact that several physical mechanisms are involved [3]; namely, diffraction, thermal effects, gain and refractive-index spectra, current distribution, etc. The initial studies of polarization instabilities in VCSELs $[4,5]$ considered the polarization dynamics in the absence of spatial effects, but the increasing evidence of the interplay of polarization and spatial effects has stimulated research incorporating this dependence $[1,6]$.

The numerical complexity of a spatiotemporal description of the VCSEL can be largely alleviated by expanding the optical field in cavity modes [7], specially for devices with a low number of confined modes. This approach has been extensively used in index-guided devices (air-post [8], etched mesa [9], or oxidized layer [10]), where methods for obtaining both scalar [11] and vectorial $[12,13]$ modes are well known. However, lateral confinement of the optical field in gain-guided devices solely relies on the spatial distribution of the gain, with the help of a generally weak, thermally induced lens [3]. Different approximate analytical methods have been proposed for determining the modal structure of these devices, e.g., the complex square-law medium [14] or variational methods [15]. However, their applicability to study large-signal dynamics in these systems is dubious be- cause the modes can be quite sensitive to the spatial distribution of the carrier density, which in turn depends on the optical pattern through spatial-hole burning. The dynamical description of the interplay between optical field and carrier density requires consideration of the full optical susceptibility of the active medium, obtained either from microscopic theories [16] or from suitable semiclassical approximations [17]. In any case, the gain and refractive-index spectra induced by the carriers, as well as their nonlinear dependence on carrier density, are required.

In this paper, we investigate the limits of validity and usefulness of a modal description of gain-guided VCSELs by analyzing the impact of the carrier-induced gain and refractive-index spectra on the stability of the transverse modes. We compare the results obtained from the spatiotemporal description and the modal expansion, under dynamical conditions. The study focuses on the off-state bounce phenomenon that, induced by carrier diffusion, appears under large-signal modulation of the current: secondary pulsations of the optical power follow the current turn-off transient [18]. We demonstrate that there exists a critical value for the thermal lensing strength above which the predictions of the modal expansion coincide with those of the full spatiotemporal model. In the opposite case the modal description yields results that deviate from those in the spatiotemporal model. The differences arise from the dynamical redistribution in carrier-induced refractive index. The paper is organized as follows: In Sec. II A we put forward the model for the spatiotemporal description of the optical properties of VCSELs. Next in Sec. II B, we perform the modal expansion of the aforementioned model. In Sec. III we perform the comparison between both models, and finally Sec. IV is devoted to summarizing and concluding the paper.

\section{THE MODEL}

\section{A. Full spatiotemporal description}

We consider the spatiotemporal model for the dynamics of the optical field in VCSELs given in Ref. [1]. This model 
describes the lateral dependence of the slowly varying amplitudes (SVA) of the electric field around the optical carrier frequency $\Omega$, expressed in the basis of circular polarization components $A_{ \pm}$. The circular components interact only with spin-up or spin-down carriers, and the two carrier densities are coupled among them by spin-flip processes. Then the model constitutes a natural generalization of the spin-flip model (SFM) [4], extensively used in the literature to describe polarization dynamics in VCSELs. For a detailed derivation of the model and a discussion of the involved physical processes, the reader is addressed to Ref. [1]. The evolution of the optical field and carrier variables is governed by

$$
\begin{aligned}
\partial_{t} A_{ \pm}\left(\vec{r}_{\perp} ; t\right)= & -\kappa A_{ \pm}+i \hat{\mathcal{L}} A_{ \pm}+i \frac{a \Gamma}{2} P_{ \pm}\left(\vec{r}_{\perp} ; t\right) \\
& -\left(\gamma_{a}+i \gamma_{p}\right) A_{\mp}+\sqrt{\beta_{s p} D_{ \pm}} \xi_{ \pm}\left(\vec{r}_{\perp} ; t\right), \\
\partial_{t} D_{ \pm}\left(\vec{r}_{\perp} ; t\right)= & \frac{\mu(t)}{2} C\left(\vec{r}_{\perp}\right)-A D_{ \pm} \\
& -\left(B N_{t}\right) D_{ \pm}^{2} \mp \gamma_{j}\left(D_{+}-D_{-}\right)+\mathcal{D} \nabla_{\perp}^{2} D_{ \pm} \\
& +\frac{a}{2 i}\left(P_{ \pm} A_{ \pm}^{*}-P_{ \pm}^{*} A_{ \pm}\right),
\end{aligned}
$$

$P_{ \pm}\left(\vec{r}_{\perp} ; t\right)$ being the SVA of the circular components of the active material polarization. $D_{ \pm}$denotes the density of spin-up (spin-down) carriers normalized to the transparency carrier density $N_{t}$.

The optical carrier frequency $\Omega$ for the SVA components of the electric field $A_{ \pm}$and the material polarization $P_{ \pm}$is that of the longitudinal mode that corresponds to an isotropic, laterally homogeneous distribution of refractive-index distribution $n_{e}$. Dichroism and birefringence of the cavity are described by $\gamma_{a}$ and $\gamma_{p}$, respectively. The deviations from the homogeneous distribution of refractive index due to either thermal lensing, an oxide layer or a mesa structure, are described by $\Delta n\left(\vec{r}_{\perp} ; \Omega\right)$ and included in Eq. (1) through the waveguide operator

$$
\hat{\mathcal{L}} A_{ \pm}=\frac{c^{2}}{2 \Omega n_{e} n_{g}}\left[\nabla_{\perp}^{2}+\left(\frac{\Omega}{c}\right)^{2} 2 n_{e} \Delta n\left(\vec{r}_{\perp} ; \Omega\right)\right] A_{ \pm},
$$

where we have assumed weak guidance, $\Delta n\left(\vec{r}_{\perp} ; \Omega\right) \ll n_{e}$ and negligible dispersion of the excess refractive index distribution.

The SVA components of the material polarization $P_{ \pm}$ have to be determined from the optical susceptibility of the material, $\chi_{ \pm}\left(\omega, D_{+}, D_{-}\right)$, to circularly polarized light. The susceptibility components describe the gain and carrierinduced refractive-index spectra of the active region, and they must be known for the model be closed, although no specific forms are needed until Sec. III. Since in the fre-

\begin{tabular}{|c|c|c|c|}
\hline Symbol & Meaning & Value & Dim. \\
\hline$a \chi_{0}$ & Effective gain constant & $1.3 \times 10^{4}$ & $\mathrm{~ns}^{-1}$ \\
\hline$\Gamma$ & Longitudinal confinement factor & 0.045 & \\
\hline$\gamma$ & Polarization decay rate & 10 & $\mathrm{ps}^{-1}$ \\
\hline$n_{e}$ & Background refractive index & 3.3 & \\
\hline$n_{g}$ & Group refractive index & 3.5 & \\
\hline$\lambda$ & Free-space wavelength & 0.85 & $\mu \mathrm{m}$ \\
\hline$\sigma$ & Band-gap shrinkage & 0.2 & \\
\hline$b$ & Empty band contribution to $\chi$ & $2 \times 10^{4}$ & \\
\hline$\kappa$ & Cavity losses & 300 & $\mathrm{~ns}^{-1}$ \\
\hline$\gamma_{a}$ & Linear dichroism & 0.5 & $\mathrm{~ns}^{-1}$ \\
\hline$\gamma_{p}$ & Linear birefringence & 30 & $\mathrm{~ns}^{-1}$ \\
\hline$A$ & Nonradiative recombination rate & 1.0 & $\mathrm{~ns}^{-1}$ \\
\hline$B$ & Bimolecular recombination rate & $10^{-11}$ & $\mathrm{~cm}^{3} \mathrm{~s}^{-1}$ \\
\hline$N_{t}$ & Transparent carrier density & $10^{18}$ & $\mathrm{~cm}^{-3}$ \\
\hline$\gamma_{j}$ & Spin-flip rate & 50 & $\mathrm{~ns}^{-1}$ \\
\hline $\mathcal{D}$ & Bimolecular diffusion & 0.5 & $\mu \mathrm{m}^{2} \mathrm{~ns}^{-1}$ \\
\hline$\beta_{s p}$ & Spontaneous emission rate & $10^{-5}$ & $\mathrm{~ns}^{-1}$ \\
\hline
\end{tabular}
quency domain we have that

$$
\widetilde{P}_{ \pm}\left(\vec{r}_{\perp} ; \nu\right)=\chi_{ \pm}\left(\Omega+\nu, D_{+}, D_{-}\right) \tilde{A}_{ \pm}\left(\vec{r}_{\perp} ; \nu\right),
$$

an approximation to its temporal evolution is [1]
TABLE I. Device and material parameters.

Then, in the above approximation the susceptibility components are evaluated at the instantaneous value of the frequency of the optical field, $\Omega_{\text {inst }}=\Omega+i \partial_{t} A_{ \pm} / A_{ \pm}$, hence the spectral changes of the gain and index are included in the dynamics.

The meaning of the remaining parameters is summarized in Table I. We consider that the shape of the transverse current density distribution is fixed by the structure of the device, so that $J\left(\vec{r}_{\perp} ; t\right)=e W N_{t} C\left(\vec{r}_{\perp}\right) \mu(t)$, where $C\left(\vec{r}_{\perp}\right)$ is the current shape and $\mu(t)$ its time dependence. Hence the total injected current reads

$$
I(t)=\mu(t) e N_{t} W \iint_{-\infty}^{\infty} C\left(\vec{r}_{\perp}\right) d^{2} \vec{r}_{\perp} .
$$

Finally, the last term in Eq. (1) models spontaneous emission processes in the semiclassical approximation. $\xi_{ \pm}\left(\vec{r}_{\perp} ; t\right)$ are random numbers with zero mean and uncorrelated in space, time, and polarization. Although noise effects are not essential for the scope of this paper, they may affect the performance of the system through switch-on time jitter, reduction of the eye diagram aperture, and mode hopping in the case of multimode operation [19].

\section{B. Modal expansion}

Obtaining the solutions of the model presented in the preceding section requires intensive computation due to the explicit spatial dependence of the optical-field components. Such an explicit dependence can be eluded, in the same way as in Refs. [7,18,20-22], by expanding the SVAs of the optical field in "cold-cavity" modes, $\Phi_{m l}$, of the transverse 
waveguide operator $\hat{\mathcal{L}}$. Then the electric field evolves accordingly with a set of ordinary differential equations for the mode amplitudes. The major inconvenience in such an approach is that the interaction with the active material has to be weak so that possible distortions of the cold-cavity modes can be neglected. In this paper, we demonstrate that the last hypothesis is not always fulfilled in gain-guided VCSELs and that there exists a minimum required lateral guiding.

The modes $\Phi_{m l}$ and the modal frequencies $\omega_{m l}$ are determined by the eigenvalue problem [11]

$$
\left[\nabla_{\perp}^{2}+\left(\frac{\Omega}{c}\right)^{2} 2 n_{e} \Delta n(r)\right] \Phi_{m l}(r, \theta)=\left(\frac{W_{m l}}{r_{g}}\right)^{2} \Phi_{m l}(r, \theta),
$$

$r_{g}$ being the waveguide radius. The modal frequencies $\omega_{m l}$, which are found from the dimensionless cladding propagation constant $W_{m l}$,

$$
\omega_{m l}=-\frac{c^{2}}{2 \Omega n_{e} n_{g}}\left(\frac{W_{m l}}{r_{g}}\right)^{2},
$$

describe the frequency redshift between the mode considered and the optical carrier frequency $\Omega$.

The solutions $\Phi_{m l}$ define a complete set of orthogonal eigenfunctions, with the mode profile having $(m-1)$ zeros in the radial direction whereas $(2 l)$ zeros occur in the angular direction. $\Phi_{10}$ is referred as the fundamental mode, $\Phi_{11}$ as the first-order transverse mode, and the remaining as higher order transverse modes. It is worth recalling that these cavity modes and modal frequencies are polarization independent, and that the cavity anisotropies that may favor one linearly polarized state over the orthogonal one have been included through the $\gamma_{a}$ and $\gamma_{p}$ parameters. We therefore express the SVA of each circular component of the field as

$$
A_{ \pm}\left(\vec{r}_{\perp} ; t\right)=\sum_{m l} \Phi_{m l}\left(\vec{r}_{\perp}\right) a_{ \pm m l}(t) e^{-i \omega_{m l} t}
$$

where $a_{ \pm m l}(t)$ stands for the complex circular components of the modal amplitude associated with the transverse mode $\Phi_{m l}$, which is normalized such that

$$
\iint\left|\Phi_{m l}\left(\vec{r}_{\perp}\right)\right|^{2} d^{2} \vec{r}_{\perp}=1
$$

In the frequency domain, Eq. (9) reads

$$
\widetilde{A}_{ \pm}\left(\vec{r}_{\perp} ; \nu\right)=\sum_{\alpha} \Phi_{\alpha}\left(\vec{r}_{\perp}\right) \tilde{a}_{ \pm \alpha}\left(\nu-\omega_{\alpha}\right),
$$

where $\alpha=\{m, l\}$ is a shorthand index for the transverse modes, with $m=0,1, \ldots$, and $l=0, \pm 1, \ldots$ By substituting it into the constitutive relationship Eq. (4), we have

$$
\widetilde{P}_{ \pm}\left(\vec{r}_{\perp}, \nu\right)=\sum_{\alpha} \chi_{ \pm}\left(\Omega+\nu, D_{+}, D_{-}\right) \tilde{a}_{ \pm \alpha}\left(\nu-\omega_{\alpha}\right) \Phi_{\alpha}\left(\vec{r}_{\perp}\right) .
$$

Since the modal amplitudes are nearly monochromatic around each modal frequency $\left[\tilde{a}_{ \pm \alpha}\left(\nu-\omega_{\alpha}\right) \neq 0\right.$ only when $\left.\nu \approx \omega_{\alpha}\right]$ we use the modal reference frames $u_{\alpha} \equiv \nu-\omega_{\alpha}$ for transforming to time domain. Using the same technique as before for performing such a step [1], we have

$$
\begin{aligned}
P_{ \pm}\left(\vec{r}_{\perp}, t\right) \approx & \sum_{\alpha}\left[\chi_{ \pm}\left(\Omega+\omega_{\alpha}+i \frac{\dot{a}_{ \pm \alpha}(t)}{a_{ \pm \alpha}(t)}, D_{+}, D_{-}\right)\right. \\
& \left.\times a_{ \pm \alpha}(t) \Phi_{\alpha}\left(\vec{r}_{\perp}\right) e^{-i \omega_{\alpha} t}\right] .
\end{aligned}
$$

Upon substitution of Eqs. (9) and (13) into the field equation (1) and by projecting onto a mode $\Phi_{\beta}^{*}\left(\vec{r}_{\perp}\right)$, we arrive at the rate equation for the amplitude of the mode considered,

$$
\dot{a}_{ \pm \beta}(t)=-\kappa a_{ \pm \beta}+\frac{i a \Gamma}{2} p_{ \pm \beta}(t)-\left(\gamma_{a}+i \gamma_{p}\right) a_{\mp \beta}+F_{\beta}(t),
$$

while by substituting Eqs. (9) and (13) into Eq. (2), we obtain

$$
\begin{aligned}
\partial_{t} D_{ \pm}\left(\vec{r}_{\perp} ; t\right)= & \frac{\mu(t)}{2} C\left(\vec{r}_{\perp}\right)-A D_{ \pm}-\left(B N_{t}\right) D_{ \pm}^{2} \\
& \mp \gamma_{j}\left(D_{+}-D_{-}\right)+\mathcal{D} \nabla_{\perp}^{2} D_{ \pm}+\frac{a}{2 i} \\
& \times \sum_{\alpha, \beta}\left[e ^ { i ( \omega _ { \beta } - \omega _ { \alpha } ) } \Phi _ { \alpha } \Phi _ { \beta } ^ { * } \chi _ { \pm } \left(\Omega+\omega_{\alpha}\right.\right. \\
& \left.\left.+i \frac{\dot{a}_{ \pm \alpha}(t)}{a_{ \pm \alpha}(t)}, D_{+}, D_{-}\right) a_{ \pm \alpha}(t) a_{ \pm \beta}^{*}(t)-\text { c.c. }\right] .
\end{aligned}
$$

Note that the stimulated recombination terms in the above equation retain the local spatial dependence of the carrier variables. On the contrary, in the modal material polarization defined through

$$
\begin{aligned}
p_{ \pm \beta}(t)= & \sum_{\alpha}\left[e ^ { i ( \omega _ { \beta } - \omega _ { \alpha } ) t } a _ { \pm \alpha } ( t ) \int \int _ { - \infty } ^ { \infty } \Phi _ { \beta } ^ { * } \chi _ { \pm } \left(\Omega+\omega_{\alpha}\right.\right. \\
& \left.\left.+i \frac{\dot{a}_{ \pm \alpha}(t)}{a_{ \pm \alpha}(t)}, D_{+}, D_{-}\right) \Phi_{\alpha} d^{2} \vec{r}_{\perp}\right],
\end{aligned}
$$

the carriers appear under an integral sign. Hence, the dependence of $p_{ \pm \beta}$ on the carrier variables has a nonlocal character. $p_{ \pm \beta}$ represents the projection of the active material polarization onto the modal profile $\Phi_{\beta}^{*}\left(\vec{r}_{\perp}\right)$, i.e., the carrierinduced gain and refractive-index change experienced by the mode. The model is thus similar to that developed in Refs. $[7,22]$, where the spatial dependence on the carrier density is explicitly maintained for the computation of the modal gains, thus including the effects of spatial-hole burning. Our description, though, includes not only self-saturation effects of 
the modal gains, but also cross-saturation effects among the modes, because the $\beta$ component of the material polarization is not diagonal in the field amplitudes $a_{\beta}$, rather it contains a superposition of different contributions arising from all the modes. By taking the usual definition of the modal gain $X_{\alpha}^{ \pm}$ of a transverse mode $\alpha$, as the imaginary part of the coefficient of the diagonal element of $p_{ \pm \beta}$,

$$
X_{\alpha}^{ \pm} \equiv-\frac{a \Gamma}{2} \iint \operatorname{Im} \chi_{ \pm}\left(\Omega+\omega_{\alpha}, D_{+}, D_{-}\right)\left|\Phi_{\alpha}\right|^{2} d^{2} \vec{r}_{\perp},
$$

it is clear that the other, nondiagonal terms in $p_{ \pm \beta}$ lead to cross saturation. Moreover, it is worth noting that the modal beat notes $\left(\omega_{\beta}-\omega_{\alpha}\right)$ appear in the evolution of the carrier densities, Eq. (15). These beat terms will be negligible when the frequency splitting between transverse modes is large enough, since in this case nonstimulated carrier recombination induces a low-pass filtering effect that strongly damps them, but not otherwise. We shall thus include in our description only the terms arising from frequency-degenerate modes and neglect the other ones.

Finally, the statistical properties of the projected Langevin noise sources $F_{ \pm \beta}(t)$ are

$$
\begin{aligned}
\left\langle F_{ \pm \beta}(t) F_{ \pm \alpha}^{*}\left(t^{\prime}\right)\right\rangle= & \delta_{+,-} \delta\left(t-t^{\prime}\right) 2 \beta_{s p} \\
& \times \iint_{-\infty}^{\infty} \Phi_{\beta}^{*}\left(\vec{r}_{\perp}\right) D_{ \pm} \Phi_{\alpha}\left(\vec{r}_{\perp}\right) d^{2} \vec{r}_{\perp},
\end{aligned}
$$

$$
\left\langle F_{ \pm \beta}(t)\right\rangle=0,
$$

indicating that, in general, correlation may exist between the spontaneous emission terms in different transverse modes due to the limited extent of the pump region.

\section{Injection and response of the active medium}

For the sake of simplicity, we assume that the radial distribution of injection current in the quantum well $(\mathrm{QW})$ plane is approximated by

$$
C(\rho)=e^{-\rho^{6}} e^{n \rho^{2}}
$$

with $\rho=r / r_{c}, r_{c}$ being the radius of the contact. When $n$ $=0$, the current distribution is super-Gaussian and describes a disk-shaped electrical contact, while a ring-shaped contact is described by $n>0$, with larger current crowding the larger $n$. In addition, a truncated parabolic waveguide

$$
\Delta n(R)= \begin{cases}\Delta n_{t l}\left[1-R^{2}\right] & \text { if } R<1 \\ 0 & \text { if } R \geqslant 1\end{cases}
$$

mimics the effect of a thermal lens. $\Delta n_{t l}$ is the thermal lensing strength, $R \equiv r / r_{g}$, and $r_{g}$ the waveguide radius.

Finally, we consider an analytical approximation to the optical susceptibility of the QW, equivalent to that given in Ref. [17], but for the circular components of the optical field.
This description captures the essential features of the gain and index spectra in QWs. The analytical expression for the optical susceptibility reads

$$
\begin{aligned}
\chi_{ \pm}( & \left.\Omega+\nu, D_{+}, D_{-}\right) \\
= & -\chi_{0}\left[\ln \left(1-\frac{2 D_{ \pm}}{u+i}\right)+\ln \left(1-\frac{D_{+}+D_{-}}{u+i}\right)\right. \\
& \left.-\ln \left(1-\frac{b}{u+i}\right)\right] .
\end{aligned}
$$

The frequency dependence is incorporated through $u \equiv \Delta$ $+\nu / \gamma+\sigma\left(D_{-}+D_{+}\right)^{1 / 3}$, where $\gamma$ is the natural width of the optical transitions, $\Delta=\left(\Omega-\omega_{g}\right) / \gamma$ measures the normalized detuning of the longitudinal mode resonance with respect to the nominal band gap, and $\sigma\left(D_{-}+D_{+}\right)^{1 / 3}$ phenomenologically describes band-gap renormalization due to Coulomb interaction between electrons and holes, $\sigma$ being the bandgap renormalization parameter.

\section{RESULTS}

In this section we perform a comparison between both methods in order to discuss the usefulness and limits of validity of a modal description of the spatiotemporal dynamics. We focus our discussion onto the study of the VCSEL dynamics during turn-on and turn-off transients accompanying the large-signal modulation.

\section{A. Single-mode operation}

As a starting point, we consider the case of a single-mode VCSEL $\left(r_{c}=r_{g}=3 \mu \mathrm{m}\right)$ with a disk-shaped contact $(n$ $=0)$ being modulated by a square-wave signal from the threshold to four times the threshold with a modulation pe$\operatorname{riod} T=10 \mathrm{~ns}$.

In Fig. 1 we plot the results obtained from direct integration of the two-dimensional (2D) model (solid lines) together with those obtained after performing the modal expansion (dashed lines) for a relatively strong thermal lens, $\Delta n_{t l}$ $=10^{-2}$. The two models yield very similar results for the dynamics of both the power and carrier density, although it can be seen that for very low power levels, there are some small differences among them due to the different way of including spontaneous emission noise in each model. During the current switch-on, the intensity and modal gain undergo typical relaxation oscillations. Following the turn-off transient, we observe the appearance of an optical pulse, generally, with low intensity levels. We explicitly define a secondary pulsation [18] as the first optical peak that follows the current turn-off. This effect is also referred as off-state bounce in optical transmission systems using digital encoding $[21,23-26]$. Since this is a detrimental effect that can affect the performance of the bit recovering process, it is imperative to have a thorough understanding and control of such a phenomenon, thus requiring fast and accurate simulation tools. Secondary pulsations can be characterized by two figures of merit, namely: the OFF/ON ratio that measures the power relation between the maximum of the first optical sec- 


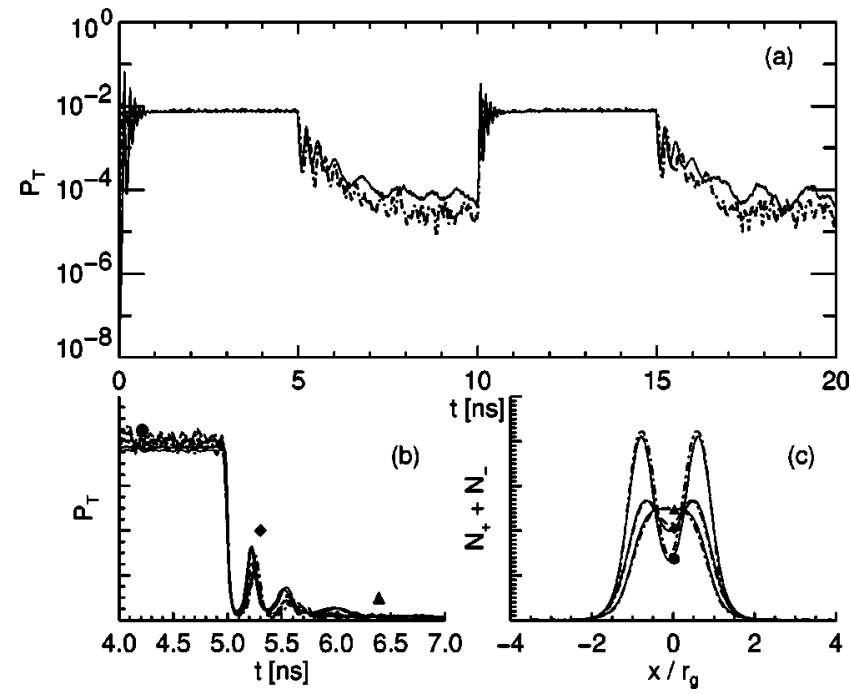

FIG. 1. Turn-on and turn-off transients for strong lateral guiding conditions. Numerical simulation of the spatiotemporal dynamics (solid lines) in comparison with the modal expanded method (dashed lines). Parameters: disk electric contact with $n=0, \mu_{b}$ $=\mu_{t h}, \mu_{o n}=4 \mu_{t h}, r_{c}=r_{g}=3 \mu \mathrm{m}, \mathcal{D}=0.5 \mu \mathrm{m}^{2} / \mathrm{ns}, \Delta n_{t l}=10^{-2}$, $\Delta=1.0$.

ondary peak with respect to the $\mathrm{cw}$ mean power, and the time $T_{\text {off }}$ elapsed between the current turn-off and the first secondary pulsation. The presence of spontaneous emission noise induces small fluctuations in these magnitudes from one bit to the other [19]. As has been already commented, these pulsations cannot be explained in terms of the typical relaxation oscillations that appears in gain-switched semiconductor lasers, since the bias current is usually set at the threshold or slightly below it. In contrast to relaxation oscillations, secondary pulsations are explained in terms of a diffusive spatial effect. When the laser switches on, the spatial-hole burning effect causes a hole in the center of the carrier distributions [see Fig. 1(c)]. When the current is turned off, the hole in the carrier distribution is filled by diffusion processes and, under appropriate conditions, provides extra gain during a short period that finally produces the optical pulses. Both models exhibit, for the parameters considered, anomalous turn-off transients with multiple secondary pulsations, whose peak power reaches about $40 \%$ of the $\mathrm{cw}$ power in both models. Also, the time at which the secondary pulses occur are almost the same in both models ( $T_{\text {off }} \approx 0.22 \mathrm{~ns}$ ), and the degree of spatial-hole burning in the carrier density is also very similar. We also find that the strength of the secondary pulsations, OFF/ON ratio, decreases both when the bias current is reduced and when the "on" current approaches the threshold in correspondence with previous studies [18,20].

Performing the same analysis for a weaker thermal lens, $\Delta n_{t l}=3 \times 10^{-3}$, the agreement between the two models becomes worse. Solid lines in Fig. 2 represent the results obtained by direct integration of the 2D model, whereas dashed lines are those obtained after performing the modal expansion. In this case, although the two models yield the same qualitative turn-off dynamics and they lead to the same cw powers, significant quantitative differences can be observed

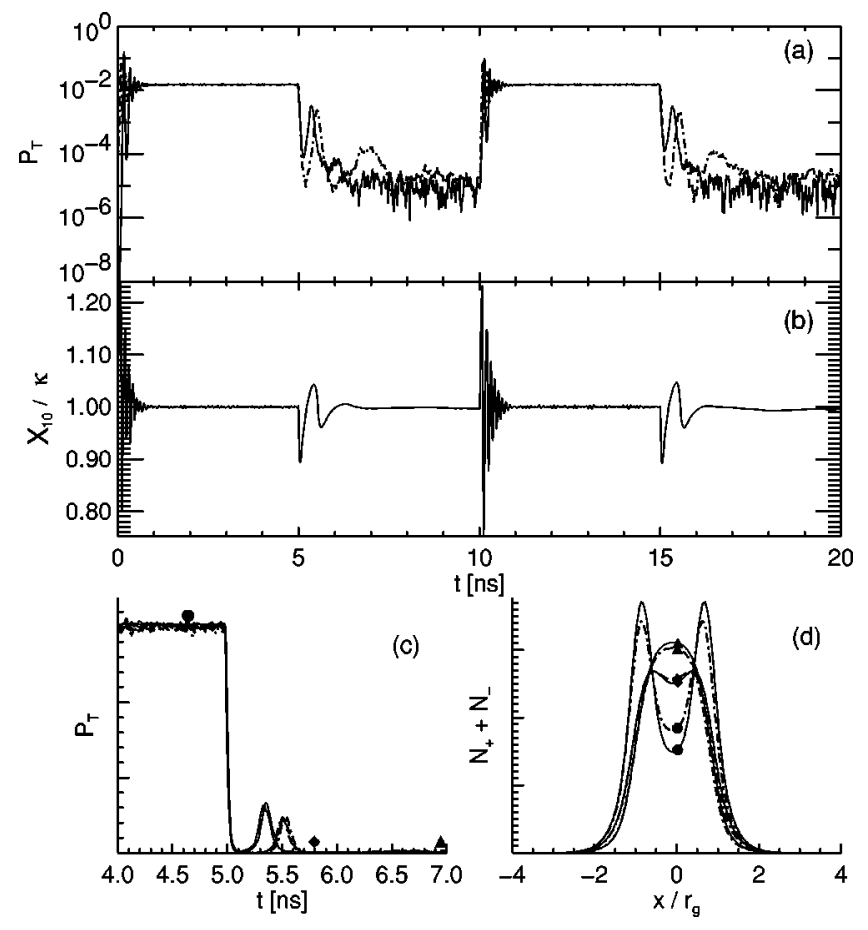

FIG. 2. Evolution of the optical intensity in log scale (a), modal gain scaled to losses (b), detail of the turn-off transients in linear scale (c), and cross section of the carrier densities at different time stages (d). The results from the 2D model are plotted in solid lines and the corresponding optical profiles are shown in Fig. 4(c). We use dashed lines for the modal expansion, except in panel (b). The secondary pulsations obtained from the $2 \mathrm{D}$ model have OFF/ON $\sim 22 \%$ and $T_{\text {off }} \sim 0.34 \mathrm{~ns}$, while $\mathrm{OFF} / \mathrm{ON} \sim 16 \%$ and $T_{\text {off }}$ $\sim 0.52 \mathrm{~ns}$ from the modal expansion. Parameters: disk electric contact with $n=0, \mu_{b}=\mu_{t h}, \quad \mu_{o n}=4 \mu_{t h}, \quad r_{c}=r_{g}=3 \mu \mathrm{m}, \quad \mathcal{D}$ $=0.5 \mu \mathrm{m}^{2} / \mathrm{ns}, \Delta n_{t l}=3 \times 10^{-3}, \Delta=1.0$.

among them during the dynamics. In particular, the ratio of the secondary pulse height to the $\mathrm{cw}$ power $(\mathrm{OFF} / \mathrm{ON})$ is significantly higher for the $2 \mathrm{D}$ model $(\approx 22 \%)$ than for the modal expansion $(\approx 16 \%$ only). Moreover, the time at which the secondary pulses appear is also significantly different for the two models: in the first, the secondary pulse appears with a delay $T_{\text {off }} \approx 0.34$ ns after the current is switched off, while in the second, the delay $T_{\text {off }} \approx 0.52 \mathrm{~ns}$. Finally, the spatial hole burnt in the carrier density is larger in the 2D model. Further reduction of $\Delta n_{t l}$ leads to larger differences between the two models.

In Fig. 3 we compare the results obtained with the two models for the same parameters as before, but in the case of a ring contact with depth $n=2$. In this case, the carrier distribution develops a marked peak at the carrier crowding radius in order to provide sufficient gain to reach lasing in the fundamental mode, and the threshold is larger than in the former case due to the smaller overlap among the Gaussian mode and the ring-shaped carrier distribution. This effect finally leads to carrier distributions with strong radial gradients. When the current is turned off, this large gradient produces a rapid filling of the hole, providing small $T_{\text {off }}$ times and rather large $\mathrm{OFF} / \mathrm{ON}$ ratios. However, the shape of the current injection does not affect the conclusions regarding the influence of $\Delta n_{t l}$ on the agreement between the two mod- 


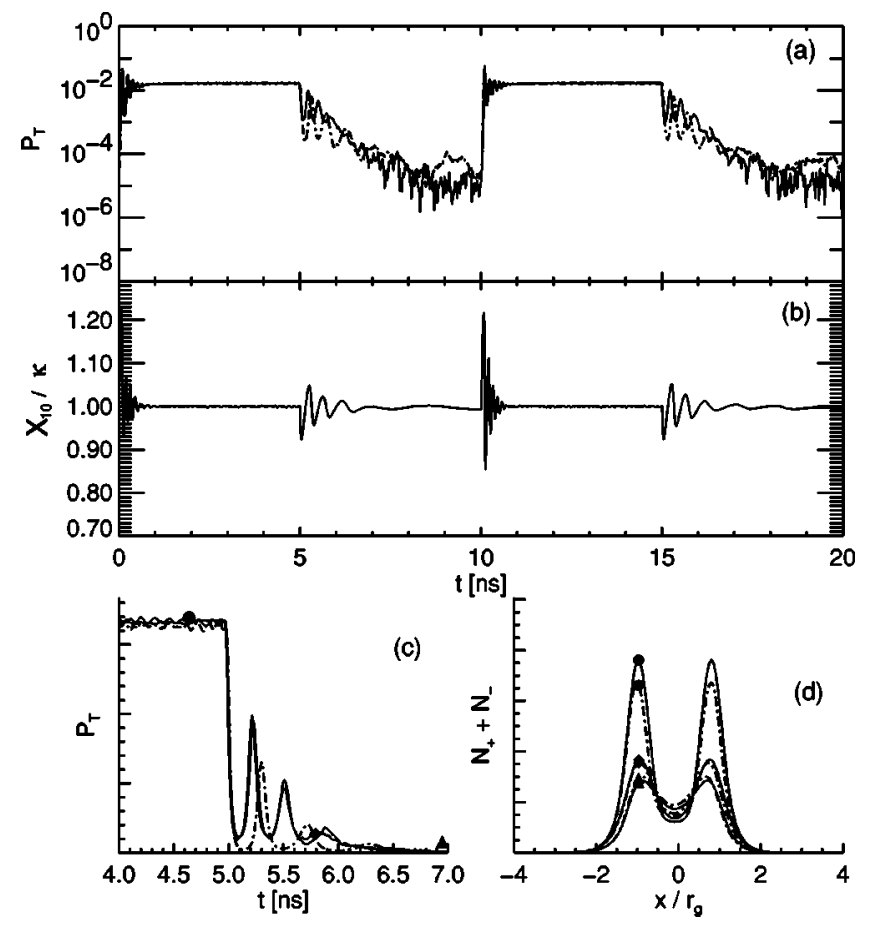

FIG. 3. Typical secondary pulsations during the turn-off transient of a VCSEL with a ring-shaped electrical contact with ( $n$ $=2$ ). Secondary pulsations from the $2 \mathrm{D}$ model $\mathrm{OFF} / \mathrm{ON} \sim 59 \%$ and $T_{\text {off }}=0.22 \mathrm{~ns}$, and from the modal expansion OFF/ON $\approx 40 \%$ and $T_{\text {off }} \approx 0.30 \mathrm{~ns}$. The remaining parameters are the same as in Fig. 2.

els: the quantitative discrepancies between the two models are even larger than for the disk-contact VCSEL and they also increase as the thermal lens weakens, although their results match for relatively large $\Delta n_{t l}$. In panels 2(b) and $3(\mathrm{~b})$, we represent the evolution of the modal gain of the fundamental mode (obtained from the modal expansion). The modal gain equals losses at the threshold, $X_{10} \approx \kappa$, which coincides with our bias current. Following the turn-on transients, we observe modal gain variations resulting from the relaxation oscillations. On the other hand, during the turn-off transients, the hole filling produces oscillations in the modal gain that finally lead to the appearance of secondary pulsations. However, as already mentioned, the spatial hole is deeper in the 2D model, which leads to an underestimation of the height and time of the secondary pulsations.

In order to clarify the origin of these discrepancies, we have analyzed how robust the modal profiles are during the switch-on and turn-off dynamics due to the changes in refractive index associated with the carrier density. Any changes in the modal profiles are naturally accounted for within the spatiotemporal description, but not in the modal expansion. In Fig. 4, we represent a cross-section of the optical profiles at different time stages. For large thermal lensing strength, the modal profiles and modal frequencies are quite robust against perturbations produced by the carrierinduced refractive index. In this limit, the modal expansion provides an accurate description of the turn-off dynamics since the modal profiles do not change. However, this is not the case when the thermal lens is weak [see Fig. 4(d)]. In this
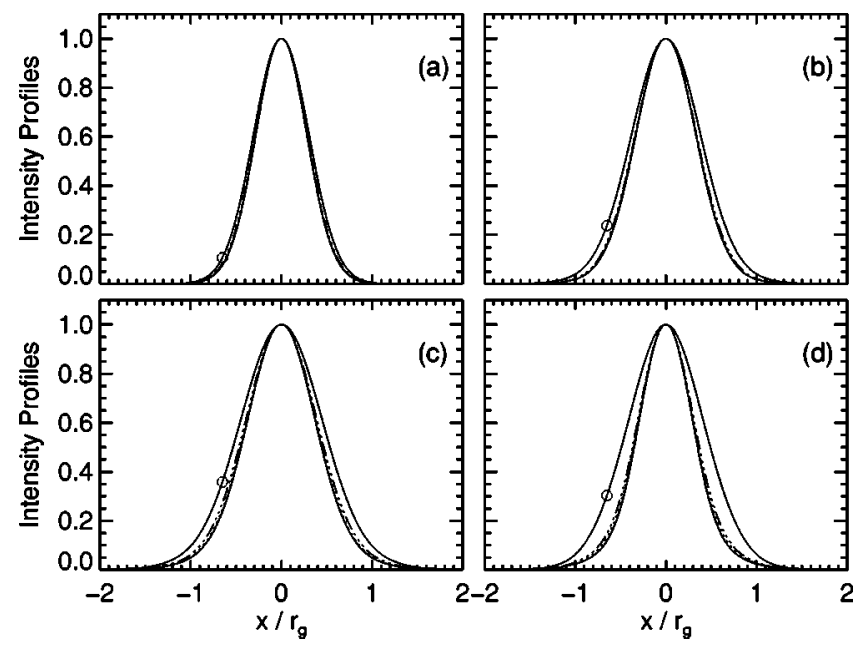

FIG. 4. Optical profiles computed from the numerical integration of the spatiotemporal dynamics at different time stages: beginning of the laser onset (solid lines and circle), under continuous wave operation (solid lines), first peak of secondary pulsations (dotted lines), and first optical valley (dash-dotted lines). The mode shrinkage is (a) $\sim 7 \%$ for $\Delta n=10^{-2}$, (b) $\sim 12 \%$ for $\Delta n=5$ $\times 10^{-3}$, (c) $\sim 17 \%$ for $\Delta n=3 \times 10^{-3}$, and (d) $\sim 30 \%$ for $\Delta n=9$ $\times 10^{-4}$.

case, following the laser switch-on, we observe a considerable mode shrinkage. When the laser switches on, it produces spatial-hole burning in the carrier distribution. Thus, the resulting distribution of refraction index is disturbed, being larger in the center than in the carrier crowding radius. This leads to a waveguide that provides an extra lateral confinement of the electric field that, in turn, is responsible for the mode shrinkage. The above observations allow to simply explain the discrepancies between the two models observed in the dynamics: for weak thermal lensing, the passive waveguide is noticeably distorted due to the carrier-induced index change, as can be seen in Fig. 5. The extra lateral confinement of the field in the 2D model due to the spatial hole burnt in the carrier density favors the activation of secondary pulsations, which are stronger and occur sooner than in the modal expansion method because in this case the field is less confined to the injection region. From this analysis we conclude that in the single-mode case the modal expansion method requires $\Delta n_{t l} \geqslant 5 \times 10^{-3}$ for having good quantitative agreement with the 2D model. Qualitatively correct results can be obtained with the modal expansion method for $\Delta n_{t l} \geqslant 3 \times 10^{-3}$. Below this value, the modal expansion approximation can no longer be used, since it leads to qualitative discrepancies with the 2D model.

\section{B. Multimode operation}

As a final example, we consider a multimode VCSEL with larger radius $r_{c}=r_{g}=4 \mu \mathrm{m}$, and moderate thermal lensing strength $\Delta n_{t l}=4 \times 10^{-3}$. So, the transverse modes involved in the dynamics are the fundamental $\mathrm{LP}_{10}$ and the first-order transverse $\mathrm{LP}_{11}$ modes in any of the two possible polarizations. In this situation, we analyze square-wave modulation of the injection current. 


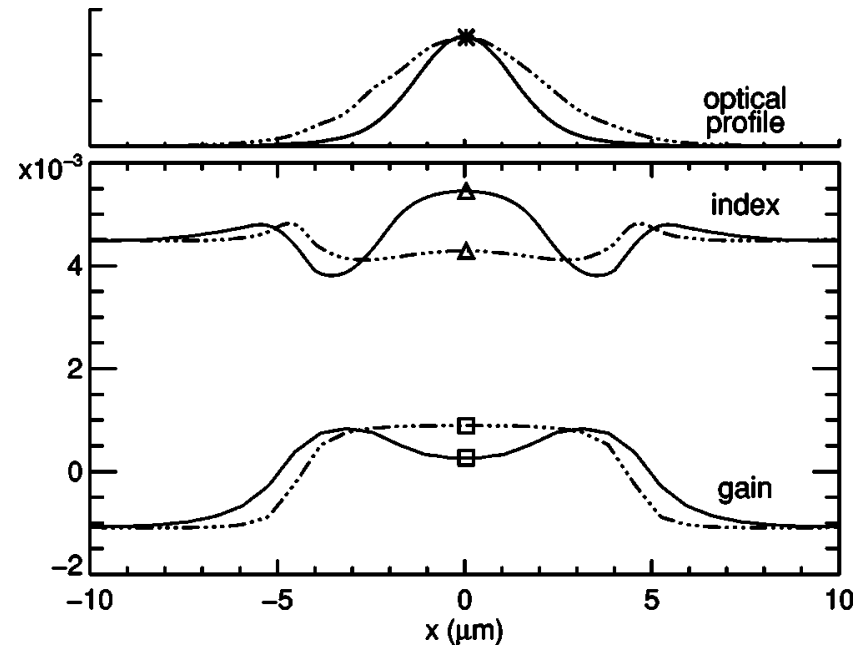

FIG. 5. Evolution of the active-material susceptibility obtained from integration of the 2D model. Cross section of $(*)$ the optical profile in arbitrary units, $(\square)$ gain $-\Gamma \operatorname{Im} \chi_{ \pm} /\left(2 n_{e}\right)$, and $(\triangle)$ total index of refraction $\Delta n(r)+\Gamma \operatorname{Re} \chi_{ \pm} /\left(2 n_{e}\right)$, in dimensionless units. Two time stages are plotted: the beginning of the laser onset (dashed lines) and cw operation (solid lines). The same parameters as in Fig. 2 except for $\Delta n_{t l}=9 \times 10^{-4}$.

The total intensity and the intensity of the secondary polarization component, obtained from the spatiotemporal model, are separately depicted in Fig. 6. We considered that the fundamental mode resonance is blueshifted with respect to the gain peak by $\Delta=1$. The general trend observed is that both polarizations initially switch on carrying significant amount of power. The secondary polarization remains active during a transient time, $\sim 1 \mathrm{~ns}$ in the figure. When the secondary polarization reaches the noise level, the total intensity undergoes additional relaxation oscillations leading to a reactivation of the secondary polarization. In order to better understand this phenomenon we have analyzed the computed near-field images, shown in Fig. 6 for certain time stages. The result is that $\sim 1 \mathrm{~ns}$ after the laser switch-on, the firstorder transverse mode starts lasing in the secondary polarization (dashed lines), and coexisting with the Gaussian mode

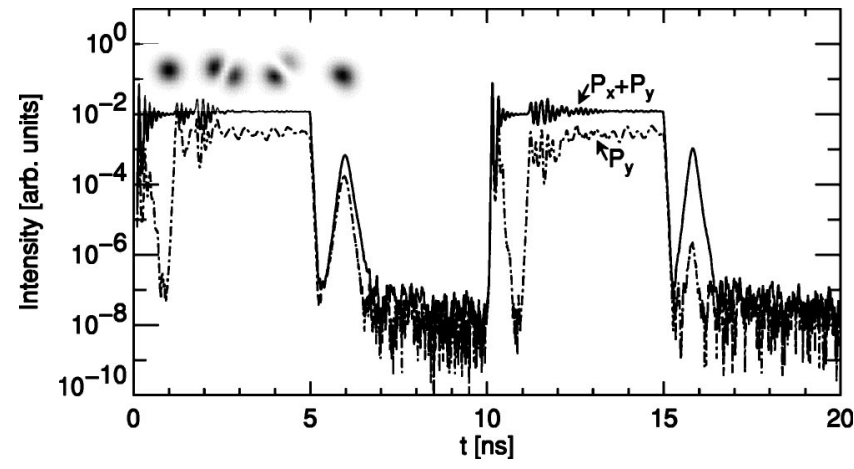

FIG. 6. Secondary pulsations in a multimode VCSEL from the full spatiotemporal model, $\mathrm{OFF} / \mathrm{ON} \approx 6 \%$. Total intensity (solid line) and secondary polarization (dashed line). Parameters: superGaussian current profile, $r_{c}=r_{g}=4 \mu \mathrm{m}, \mu_{\text {bias }}=\mu_{t h}, \mu_{o n}=4 \mu_{t h}$, $\Delta n_{t l}=4 \times 10^{-3}$, and $\Delta=1.0$.

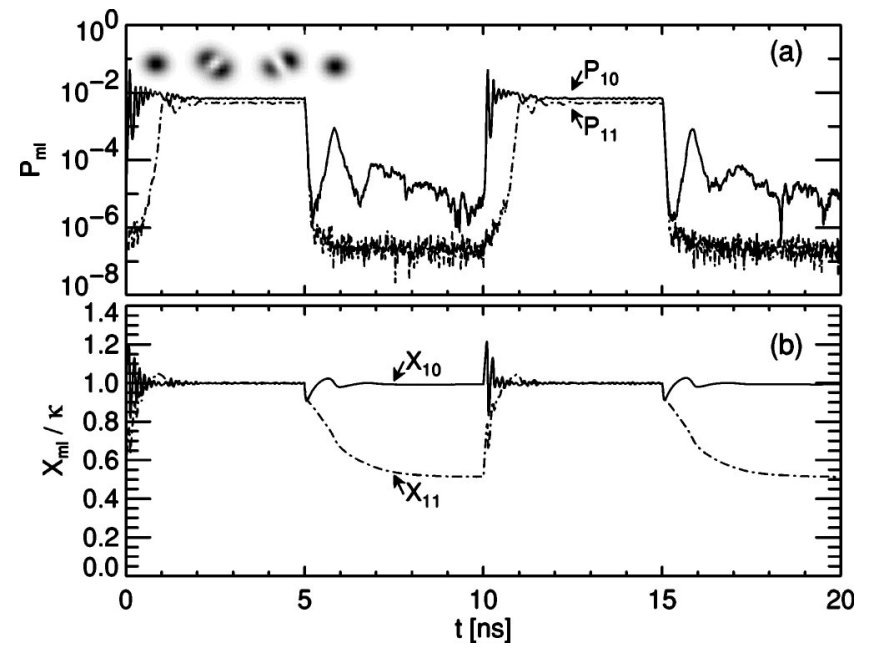

FIG. 7. Secondary pulsations in a multimode VCSEL from the modal expansion. The same parameters as in Fig. 6. Power of the $\mathrm{LP}_{10}$ mode (solid line) and power of the $\mathrm{LP}_{11}$ mode (dashed line).

until the current is turned off. By analyzing the evolution of the near-field images in the dominant polarization, we observe that the orientation of the $\mathrm{LP}_{11}$ is not fixed, but it rotates between the cos and sin modes. Following the current turn-off, we find that secondary pulsations take place in the fundamental transverse mode and that both linear polarizations carry significant amount of power. Even though the role of higher-order transverse modes is to suppress the magnitude of secondary pulsations [20], we can still observe an $\mathrm{OFF} / \mathrm{ON} \approx 6 \%$.

The modal expansion allow us to easily follow the separate evolution of the transverse-mode amplitudes $\mathrm{LP}_{10}$ and LP $_{11}$ [Fig. 7(a)]. The fundamental transverse mode is initially selected. Then, as a consequence of the global increase in carrier density and due to the spatial-hole burning in the carrier distribution, the first-order transverse mode $\mathrm{LP}_{11}$ can profit from the available material gain. The first-order transverse mode switches on at $t \sim 1 \mathrm{~ns}$, causing additional relaxation oscillations. This mode coexists with the fundamental mode until the current is turned off. Secondary pulsations appear in the fundamental mode and they have similar OFF/ON as those obtained with the spatiotemporal model. In Fig. 7(b), we represent the evolution of the modal gain for both transverse modes. During the current turn-off the $X_{10}$ modal gain exceeds the losses during a short period, which in turn originates the appearance of the secondary pulsation. However, the monotonic decrease in modal gain of $X_{11}$ is worth noting: when the current is turned off, there is a sudden interruption in carrier injection at the edges of the distribution that produces a worse overlap with the mode $\mathrm{LP}_{11}$ and thus a decrease in modal gain. The rotation of the $\mathrm{LP}_{11}$ mode is also found in the modal expansion, resulting from the cross-saturation terms between the two degenerated firstorder transverse modes. The reconstructed near-field images in Fig. 7(a) are in good agreement with those computed from the full spatiotemporal model.

\section{CONCLUSIONS}

The usefulness and limits of validity of a modal expansion for analyzing large-signal dynamics in gain-guided VC- 
SELs have been investigated by comparing the predictions of the modal expansion method with those of a full spatiotemporal description of the system. The modal expansion presented in this paper generalizes previous models by incorporating polarization effects and gain cross-saturation terms among degenerate transverse modes. The comparison has mainly focused on the secondary pulsations that appear under square-wave modulation of the injection current. We have found that the modal expansion yields qualitatively correct results for thermal lensing strengths above $\Delta n_{t l} \sim 3$ $\times 10^{-3}$. However, quantitative agreement between the two models requires $\Delta n_{t l}>5 \times 10^{-3}$ independently of the shape of the current injection. This value of $\Delta n_{t l}$ is about one order of magnitude larger than the contribution arising from the carrier-induced refractive index, thus confirming that the discrepancies originate from the fact that the modal expansion neglects the effects of the carrier-induced index of refraction on the modal profiles and frequencies: for weak lateral guiding, the mode profiles significantly change during the evolution. Furthermore, we conclude that the practical applicability of the modal expansion is limited to small VCSELs where a reduced number of transverse modes is involved. Otherwise, the computational times required for accounting for such a large number of modes is nearly equivalent to that when directly dealing with the full spatiotemporal model.

\section{ACKNOWLEDGMENTS}

This work was funded by the European Commission through the VISTA HP-TRN and the Spanish MCyT under Project Nos. TIC99-0645-C05-02 and BFM2000-1108. The authors wish to thank A. Valle and L. Pesquera for helpful discussions.
[1] J. Mulet and S. Balle, IEEE J. Quantum Electron. 38, 291 (2002).

[2] M. San Miguel, in Semiconductor Quantum Optoelectronics (Institute of Physics, Bristol, 1999), p. 339; K. J. Ebeling, ibid., p. 295.

[3] W. Nakwaski and M. Osinski, Prog. Opt. 38, 165 (1998).

[4] M. San Miguel, Q. Feng, and J.V. Moloney, Phys. Rev. A 52, 1728 (1995).

[5] J. Martín-Regalado, F. Prati, M. San Miguel, and N.B. Abraham, IEEE J. Quantum Electron. 33, 765 (1997).

[6] D. Burak, J.V. Moloney, and R. Binder, IEEE J. Quantum Electron. 36, 956 (2000).

[7] A. Valle, J. Sarma, and K.A. Shore, IEEE J. Quantum Electron. 31, 1423 (1995).

[8] J.L. Jewell, A. Sherer, S.L. McCall, Y.H. Lee, S. Walker, J.P. Harbison, and L.T. Florez, Electron. Lett. 25, 1123 (1989); D. Burak and R. Binder, IEEE J. Quantum Electron. 33, 1205 (1997)

[9] W.W. Chow, K.D. Choquette, M.H. Crawford, K.L. Lear, and G.R. Hadley, IEEE J. Quantum Electron. 33, 1810 (1997).

[10] D.L. Huffaker, D.G. Deppe, K. Kumar, and T.J. Rogers, Appl. Phys. Lett. 65, 97 (1994).

[11] A. W. Synder and J. D. Love, Optical Waveguide Theory (Chapman and Hall, New York, 1983).

[12] G.P. Bava, P. Debernardi, and L. Fratta, Phys. Rev. A 63, 023816 (2001).
[13] M.J. Noble, J.P. Loehr, and J.A. Lott, IEEE J. Quantum Electron. 34, 1890 (1998).

[14] W. Nakwaski and R.P. Sarzala, Opt. Commun. 148, 63 (1998).

[15] S. Riyopoulos, D. Dialetis, J. Inman, and A. Phillips, J. Opt. Soc. Am. B 18, 1268 (2001).

[16] D. Burak, J.V. Moloney, and R. Binder, Phys. Rev. A 61, 053809 (2000).

[17] S. Balle, Phys. Rev. A 57, 1304 (1998).

[18] A. Valle, J. Sarma, and K.A. Shore, J. Opt. Soc. Am. B 12, 1741 (1995).

[19] A. Valle, J. Mulet, L. Pesquera, and S. Balle, Proc. SPIE 4649, 50 (2002).

[20] A. Valle and L. Pesquera, Appl. Phys. Lett. 79, 3914 (2001).

[21] J.J. Morikuni, P.V. Mena, A.V. Harton, K.W. Wyatt, and S.-M. Kang, J. Lightwave Technol. 17, 95 (1999).

[22] J.S. Gustavsson, J.A. Vukusic, J. Bengtsson, and A. Larsson, IEEE J. Quantum Electron. 38, 203 (2002).

[23] J. Tatum, D. Smith, J. Guenter, and R. Johnson, Proc. SPIE 3004, 151 (1997).

[24] J.A. Lehman and R.A. Morgan, in Vertical-Cavity SurfaceEmitting Lasers: Technology and Applications, edited by J. Cheng and N.K. Dutta (Gordon and Breach, Amsterdam, 2000), Chap. 3, p. 133.

[25] M.I. Cohen, A.A. Allerman, K.D. Choquette, and C. Jagadish, IEEE Photonics Technol. Lett. 13, 544 (2001).

[26] See, for instance, http://content.honeywell.com/vcsel/ technical/006703_1.pdf 